\title{
Thomas/Putzo, Zivilprozeßordnung
}

\author{
Inhaltsübersicht
}

\author{
A. Entstehung \\ B. Konzept \\ C. Der weitere Weg des „Thomas/Putzo“ \\ D. Die neuen Mitautoren \\ E. Wie geht's weiter?
}

\section{A. Entstehung}

Für mich Nachgeborenen ist es nicht ganz einfach, über die Entstehung des Kommentars zu berichten. Grundlage sind leider nicht eigene Erinnerungen, sondern Erzählungen von Hans Putzo und schriftliche Quellen aus dem Archiv des Verlages und den Vorworten zu den bisherigen Auflagen.

Für die Entstehung eines neuen juristischen Kommentars zu einem Gesetz scheint es keinen allgemein gültigen Weg zu geben. Bei belletristischen Werken sucht sich in der Regel der Autor einen Verleger, der bereit ist, das neue Werk herauszubringen. Das dürfte bei einem juristischen Kommentar kaum je geschehen. Denn welcher Jurist würde sich der Knochenarbeit des Kommentierens unterziehen, ohne vorher sicher zu sein, daß sein Werk auch gedruckt wird? Ein juristischer Kommentar entsteht deshalb gewöhnlich nur dann, wenn vorher Autor und Verleger zusammengefunden haben. Ein solches Zusammenfinden kann das Ergebnis gezielter Suche sein, aber auch durch zufällige Begegnungen und Umstände zustandekommen.

Als die Idee zu einem neuen kurzen Kommentar zur Zivilprozeßordnung Ende der fünfziger Jahre des letzten Jahrhunderts geboren wurde, war die Landschaft der ZPO-Kommentare sehr übersichtlich. Den Platz des knappen Praktiker-Kommentars nahm der „Baumbach/Lauterbach“ ein, der Anfang 1958 in der 25. (Jubiläums-)Auflage erschienen war. Eine ausführlichere Kommentierung bot der „Z̈̈ller“ (9. Aufl. 1958). Den Typus des wissenschaftlichen Kommentars verkörperte der „Stein/Jonas“, der damals als Loseblattwerk von Pohle bearbeitet wurde. Schließlich existierte noch der 6-bändige Großkommentar von Wieczorek.

Als das Manuskript für die 26. Auflage des „Baumbach/Lauterbach“, aus welchen Gründen auch immer, ungebührlich lange auf sich warten ließ, entstand im Verlag C.H. Beck die Befürchtung, ein anderer juristischer Verlag könne mit einem neuen Kurzkommentar die Lücke ausfüllen. So machte man sich von Seiten des Beck-Verlags auf die Suche nach einem geeigneten Autor.

Heinz Thomas, damals Landgerichtsrat und hauptamtlicher Leiter einer Arbeitsgemeinschaft für Rechtsreferendare, hatte in der Münchner Juristengesellschaft 
Hans-Ulrich Büchting, einen Lektor des Beck-Verlags, kennengelernt. Über den künftigen Verlagsinhaber Dr. Hans-Dieter Beck, der in München Rechtsreferendar gewesen war, erfuhr Hans-Ulrich Büchting davon, daß Heinz Thomas auch einen erfolgreichen privaten Klausurenkurs für Rechtsreferendare abhielt. Die dadurch bewiesene pädagogische Begabung empfahl Heinz Thomas als geeigneten Autor für den neuen Kurzkommentar zur ZPO. Die Anfrage von Hans-Ulrich Büchting bei ihm fiel auf fruchtbaren Boden. Allerdings wollte sich Heinz Thomas der immensen Arbeit, einen neuen Kommentar zur ZPO aus dem Boden zu stampfen, nicht allein unterziehen. Parallel $\mathrm{zu}$ ihm war der etwas jüngere Hans Putzo auch zum hauptamtlichen Arbeitsgemeinschaftsleiter bestellt worden. In den gemeinsamen Vorbesprechungen der Arbeitsgemeinschaftsleiter stellten die beiden fest, daß sie didaktisch auf gleicher Wellenlänge lagen. Als deshalb Heinz Thomas im Februar 1960 Hans Putzo fragte, ob er an einem didaktisch ausgerichteten neuen ZPOKommentar mitarbeiten wolle, war dieser sofort dazu bereit. Nach Absprache mit Hans-Ulrich Büchting und dem damaligen Cheflektor Hoeller lieferten sie bereits im März 1960 Probekommentierungen zu $§ 313$ und zu $§ 767 \mathrm{ab}$, die im Verlag auf Anhieb gefielen. Im Mai 1960 wurden die Verlagsverträge mit Dr. Heinrich Beck geschlossen. Geplant war eine Zeit von 21/2 Jahren für die Erstellung des Manuskripts. Zum großen Erstaunen der Mitarbeiter im Verlag lieferten die beiden Autoren das Manuskript bereits nach 21/4 Jahren ab.

Im Herbst 1962 ging das Werk in Druck und im März 1963 erschien die erste Auflage als erläuterte Ausgabe der ZPO mit einem Umfang von 1126 Seiten in 5000 Exemplaren. Der Preis war mit DM 28,50 festgesetzt und betrug damit kaum mehr als die Hälfte des Preises des Kommentars von Baumbach. Dessen Autor Dr. Wolfgang Lauterbach, Senatspräsident am Hanseatischen Oberlandesgericht Hamburg, war über das Erscheinen eines Konkurrenzprodukts im selben Verlag äußerst erbost und suchte eifrig nach „Plagiaten“, konnte aber keine finden.

\section{B. Konzept}

Der neue Kommentar sollte handlich und für den täglichen Gebrauch bestimmt sein. Er erschien deshalb in der gerade entstehenden Gelben Reihe im Taschenformat mit einem Umfang von weniger als 1500 Seiten. Die Erläuterungen sollten sich auf das Wesentliche beschränken und möglichst die Ansicht der höchstrichterlichen Rechtsprechung oder der herrschenden Meinung darstellen. Für vertiefte wissenschaftliche Auseinandersetzung blieb dabei ebensowenig Raum wie für die Bewertung von Gesetzesänderungen. Die prozessualen Grundbegriffe und die Prozeßmaximen wurden in der Einleitung abgehandelt, Begriffsbestimmungen und paragraphenübergreifende Erklärungen in Vorbemerkungen übersichtlich und systematisch dargestellt. Die Kommentierungen der einzelnen Vorschriften wurden nach einem einheitlichen Aufbau gestaltet und übersichtlich gegliedert. Das sollte die schnelle Orientierung und das rasche Auffinden der gesuchten Antwort erleichtern.

Das Buch richtete sich nicht nur an die Praktiker in Anwaltschaft und den Gerichten, sondern vor allem auch an die Jurastudenten, Rechtsreferendare und 
angehende Rechtspfleger. Es sollte also auch denjenigen die Anwendung der Zivilprozeßordnung ermöglichen, die davon noch keine praktischen Anschauungen hatten. Der „Baumbach/Lauterbach“ setzte demgegenüber doch schon einige Kenntnisse des Zivilprozeßrechts voraus.

Kurz zusammenfassen läßt sich die Konzeption mit dem Vorwort zur 8. Auflage: „Praktische Systematik und übersichtliche Gliederung, umfassende Darstellung aller für Praxis, Ausbildung und Studium wichtigen Teile; Aktualität durch Wiedergabe neuer Belegstellen mit geeigneten weiteren Nachweisen."

Die Aufteilung des Stoffs wurde zwischen den beiden Autoren nach praktischen Gesichtspunkten abgesprochen, also danach, in welchem Teilgebiet der jeweilige Autor am meisten praktische Erfahrungen gesammelt hatte. Da Hans Putzo in einer Beschwerdekammer tätig gewesen war, übernahm er das Zwangsvollstreckungsrecht, während Heinz Thomas als früheres Mitglied einer erstinstanzlichen Zivilkammer den Großteil der Vorschriften über das landgerichtliche Verfahren bearbeitete. Da die beiden Autoren die Erstmanuskripte laufend austauschten und auch später ihre Kommentierungen inhaltlich abstimmten, dabei insbesondere zahlreiche Querverweisungen einfügten, entstand ein Werk aus einem Guß, so $\mathrm{da}$ es nicht erforderlich erschien, die vom jeweiligen Autor bearbeiteten Teile zu kennzeichnen. Dies geschah dann erst ab der Beteiligung weiterer Autoren.

Die zu Anfang festgelegte Aufgabenteilung war freilich keine starre, sondern wurde bei gegebenem Anlaß neu abgestimmt. So übernahm ich mit dem Inkrafttreten des ZPO-Reformgesetzes von Hans Putzo den Abschnitt über die Beschwerde und Rechtsbeschwerde als kleinen Ausgleich für die von Rainer Hüß tege bearbeiteten europarechtlichen Vorschriften, die grundsätzlich zum Arbeitsbereich von Hans Putzo gehört hätten. Mit der 27. Auflage übernahm Rainer Hüßtege die Kommentierung des $\S 323$, da er als Vorsitzender eines Familiensenats in der Praxis damit befaßt werden sollte, während ich die Verantwortung für die gesamte Einleitung übernommen habe.

\section{Der weitere Weg des „Thomas/Putzo“}

Daß der neue Kommentar von Thomas und Putzo in kurzer Zeit ein durchschlagender verlegerischer Erfolg wurde - seit der achten Auflage liegt die Höhe stets im fünfstelligen Zahlenbereich -, lag vor allem an der konsequenten Umsetzung des dargestellten Konzepts, das die didaktischen Erfahrungen der beiden Autoren verwertete und die Bedürfnisse der angehenden Juristen aufs Beste befriedigte. Ich erinnere mich noch gut, daß mir gegen Ende meines Studiums ein älterer Kollege begeistert von der Neuerscheinung und darüber berichtete, daß er anhand des neuen Werks erstmals die Grundsätze der Erledigung der Hauptsache verstanden habe.

Wie schwer es ist, den Erfolg eines neuen juristischen Werks einzuschätzen, zeigt die im Verlag auch heute noch öfters zitierte Äußerung des damaligen Lektors gegenüber den beiden Autoren: ,Wenn nach zwei bis drei Jahren die Hälfte der Erstauflage verkauft sein sollte, werden wir den Rest halt einstampfen.“ Nach 
zweieinhalb Jahren war aber nicht nur die Hälfte der Erstauflage verkauft, sondern die gesamte. Im November 1965 erschien deshalb bereits die zweite Auflage, zwei Jahre später die dritte.

Zur weiten Verbreitung des Kommentars in kurzer Zeit trug sicher auch die maßvolle Preiskalkulation des Verlegers bei, der einer eher hohen Auflage zu niedrigem Preis den Vorzug vor einer hochpreisigen Kleinauflage gab. Ein weiterer Grund für den raschen Erfolg mag auch gewesen sein, daß der „Baumbach/ Lauterbach“ in Umfang und Preis über einen Kurzkommentar hinausgewachsen war und der ,Zöller“ viele Jahre nicht in einer Neubearbeitung erschien (10. Auflage erst 1968).

Der Zeitpunkt für das Erscheinen einer Neuauflage wurde und wird anders als beim „Palandt“ nicht durch einen festen Rhythmus, sondern entweder durch den Ausverkauf der Vorauflage oder, weit häufiger, durch die Aktivitäten des Gesetzgebers bestimmt. Wie ein roter Faden zieht sich durch die Vorworte die Aufzählung der Gesetzesänderungen, die eine Neuauflage notwendig machten.

Besondere Erwähnung verdienen in diesem Zusammenhang die 9. Auflage, die - im Februar 1977 erschienen - die zum 1. Juli 1977 in Kraft tretende Vereinfachungsnovelle sowie das 1 . Ehe- und Familien-Reformgesetz zu erläutern hatte und eine Umfangserweiterung von mehr als 200 Seiten mit sich brachte, ferner die 17., die 18. und die 24. Auflage. Die 17. Auflage vom August 1991, nur zehn Monate nach der 16. erschienen, behandelte u. a. das Übergangsrecht für die neuen Bundesländer, das Rechtspflegevereinfachungsgesetz und die Änderungen des Mahnverfahrens durch das Verbraucherkreditgesetz. Die im März 1993 erschienene 18. Auflage verarbeitete vor allem das Gesetz zur Entlastung der Rechtspflege. Diese Auflage wurde auf ein größeres Format umgestellt; der Text wurde mit Randnummern versehen. Die 24. Auflage schließlich brachte die Bearbeitung des zum 1. Januar 2002 in Kraft getretenen ZPO-Reformgesetzes. Angesichts der zahllosen und tiefgreifenden Änderungen konnte sie erst im Februar 2002 in den Handel gelangen, immerhin als erster unter den ZPO-Kommentaren.

\section{Die neuen Mitautoren}

Mit 75 Jahren fand Heinz Thomas es an der Zeit, nach einem Nachfolger Ausschau $\mathrm{zu}$ halten. Ich hatte das Glück gehabt, mit 39 Jahren an das Oberlandesgericht München berufen und dem Senat zugeteilt zu werden, den Heinz Thomas bis zu seinem Eintritt in den Ruhestand leitete. Es waren für mich besonders lehrreiche und auch menschlich bereichernde Jahre, während denen ich zusätzlich nebenamtlicher Arbeitsgemeinschaftsleiter für Rechtsreferendare war. Offenbar hielt mich Heinz Thomas für geeignet, Stück für Stück an seiner Stelle die Arbeit am Thomas-Putzo zu übernehmen. Ich war glücklich über sein Angebot und das Vertrauen, das er in mich setzte. Nach einer Zwischenstation am Bayerischen Obersten Landesgericht war ich inzwischen Vorsitzender Richter am Oberlandesgericht München geworden. Wenige Wochen nach dem Neubeginn am Oberlandesgericht wurde meinem Senat Dr. Rainer Hüßtege als Richter zugeteilt, der die Jahre 
zuvor als hauptamtlicher Arbeitsgemeinschaftsleiter für Rechtsreferendare tätig gewesen war und als Schüler von Professor Erik Jayme ein ausgewiesener Kenner des internationalen Privatrechts sowie des Europarechts ist.

Da für den „Thomas/Putzo“ eine knappe Kommentierung des EuGVÜ erforderlich wurde, schlug ich Rainer Hüßtege für diese Aufgabe vor. Diese Wahl erwies sich als so glücklich, daß Hans Putzo ihm die sukzessive Nachfolge angeboten hat.

\section{E. Wie geht's weiter?}

Die Zivilprozeßordnung scheint zur Experimentierwiese und damit zur Dauerbaustelle des Gesetzgebers geworden zu sein. So werden die Autoren der Kommentare zu diesem Gesetz von Auflage zu Auflage gehetzt. Das Bemühen um Aktualität stößt sich mit dem Bestreben, dem Benutzer eine Auflage für längere Zeit anzubieten. Als die 25. Auflage, die vor allem die erste Welle der Gerichtsentscheidungen zum ZPO-Reformgesetz verarbeitete, im wesentlichen verkauft war, schied ein Nachdruck aus, weil das Kostenrechtsmodernisierungsgesetz erlassen und ein neues Buch 11 der ZPO angefügt wurde. Andererseits konnte nicht zugewartet werden, bis wir das 1. Justizmodernisierungsgesetz einarbeiten konnten. Für die 27. Auflage, die im Herbst 2005 in nochmals vergrößertem Format erschienen ist, war nicht nur das 1. Justizmodernisierungsgesetz und leider auch das Gesetz zur Auflösung des Bayerischen Obersten Landesgerichts in den Kommentar einzuarbeiten, sondern es galt auch, die neue Eheverordnung der EU und die deutschen Ausführungsvorschriften dazu ebenso zu kommentieren wie die Verfahrensvorschriften des Justizkommunikationsgesetzes. Mit der 27. Auflage hat leider Hans Putzo im 80. Lebensjahr die juristische Feder aus der Hand gelegt. Wir verbleibenden Autoren bedauern das zutiefst, können die Gründe dafür aber auch verstehen. Immerhin hat uns Hans Putzo weiterhin seinen guten Rat angeboten, der auf der Erfahrung eines langen Lebens beruht und der uns sehr nützlich sein wird. Generationen von Juristen schulden ihm großen Dank für seine beispielhaften Beiträge im ,,Thomas/ Putzo“ und im „Palandt“. Wir werden uns bemühen, den Kommentar nach dem bewährten Konzept von Heinz Thomas und Hans Putzo weiterzuführen.

Für die bevorstehende 28. Auflage kann der Gesetzestext der endlich im Dezember 2005 erstellten Neubekanntmachung der Zivilprozeßordnung entnommen werden. Außerdem ist das 2. Justizmodernisierungsgesetz einzuarbeiten, ferner sind einige kleinere Gesetzesänderungen durch das Gesetz über Kapitalanleger-Musterverfahren, das Gesetz zur Stärkung der Selbstverwaltung der Rechtsanwaltschaft, das Gesetz zur Änderung des Wohnungseigentumsgesetzes u.a. zu berücksichtigen. Weiterhin ungewiß ist das Schicksal des Entwurfs für ein Forderungssicherungsgesetz, der einige wesentliche Änderungen der ZPO vorsieht. Umfangreichere Änderungen für fernere Neuauflagen sind zu erwarten durch die geplante Reform des Unterhaltsrechts, eine umfangreiche Novelle zum Zwangsvollstreckungsrecht und das neue Gesetz über das Verfahren der freiwilligen Gerichtsbarkeit sowie durch weitere Verordnungen und Richtlinien des europäischen Gesetzgebers. 
Zudem ist es an der Zeit, energisch zu versuchen, ein weiteres Wachsen des Buchumfangs zu verhindern, im Gegenteil möglichst eine Reduzierung zu erreichen. Das wird jedenfalls mühevoller werden als einfach neuen Stoff und neue Rechtsprechung einzufuigen. Ein Trost bei dieser hektischen Gesetzgebungstätigkeit ist einmal die tatkräftige Unterstützung durch unsere Lektorin Frau Dr. Christiane Dobring, die schon vor Jahren den Arbeitsbereich von Büchting übernommen hat, und zum anderen der Umstand, daß wir Co-Autoren durch eine fast freundschaftliche Verbundenheit mit der gegenseitigen Abstimmung keine Schwierigkeiten haben - keine Selbstverständlichkeit bei selbstbewußten Juristen.

Müssen wir uns vor dem Erscheinen eines weiteren Konkurrenten fürchten?

Sicher ist das Bessere der Feind des Guten; andererseits zeigen die Beispiele des mittleren Kommentars von Musielak und des kleineren von Zimmermann, wie schwer es ist, trotz hochstehender inhaltlicher Qualität auf dem stark besetzten Feld der ZPO-Kommentare Boden zu gewinnen. Auch der neue Stern am ZPOHimmel, der von Ingo Saenger herausgegebene und mitverfaßte Handkommentar zur ZPO, wird es trotz seiner Qualitäten nicht leicht haben, sich auf dem Markt zu behaupten. 